\title{
RECURRENT, NONEQUILIBRIUM SYSTEMS AND THE MARKOV BLANKET ASSUMPTION
}

\section{A PREPRINT}

\author{
Miguel Aguilera \\ School of Engineering and Informatics \\ University of Sussex \\ Falmer, Brighton, BN1 9QJ, United Kingdom \\ sci@maguilera.net
}

\author{
Christopher L. Buckley \\ School of Engineering and Informatics \\ University of Sussex \\ Falmer, Brighton, BN1 9QJ, United Kingdom \\ C.L.Buckley@sussex.ac.uk
}

January 18, 2022

This paper is a commentary on Bruineberg, J., Dolega, K., Dewhurst, J., and Baltieri, M. (2021). The Emperor's New Markov Blankets. Behavioral and Brain Sciences. https://doi .org/10.1017/S0140525X21002351

\begin{abstract}
Markov blankets -statistical independences between system and environment- have become popular to describe the boundaries of living systems under Bayesian views of cognition. The intuition behind Markov blankets originates from considering acyclic, atemporal networks. In contrast, living systems display recurrent, nonequilibrium interactions that generate pervasive couplings between system and environment, making Markov blankets highly unusual and restricted to particular cases.
\end{abstract}

In the target article, Bruineberg et al. (2021) disrupt current debates about the role of Markov Blankets in demarcating the boundaries between living system and their environment. The authors accurately describe the gap between a Markov blanket as a useful property for statistical inference and the more ontologically loaded concept in the FEP, as a boundary within which Bayesian inference occurs. While the arguments pursued by the target article are both correct and important, we think that a fundamental concern remains unaddressed as the paper tacitly accepts (as generally the FEP does) that Markov blankets can be identified largely on the basis of the structural connectivity of a system (as opposed to its functional connectivity).

A Markov blanket is defined as a set of variables (the 'blanket') that separates two other sets of variables within a system, rendering them conditionally independent. That is, if the state of the blanket is fixed, the one set of variables (e.g., an agent) becomes independent of the second set (e.g. the environment). This property, also known as the global Markov condition (Richardson et al. 1996), depends on the (conditional) functional couplings describing the statistical interdependencies of a system. Markov blankets were initially introduced in the context of Bayesian networks (Pearl, 1988), which take the form of directed acyclic graphs. In such acyclic models, Markov blankets can be directly identified by applying a simple rule to the structural connectivity alone (e.g. Fig. 11A). In particular, the Markov blanket of a set of nodes $x$ contains the parent nodes of $x$, the children nodes of $x$ and the parents of each child (where children and parents of $x$ are defined as the nodes with incoming/outgoing connections from/to $x$ ). This specific sparse structural connectivity is defined as the local Markov condition (Richardson et al., 1996).

The FEP derives much of its intuitions about Markov blankets from acyclic models. However, the theory takes the idea much further, philosophically and mathematically. The FEP often considers the local Markov condition sufficient for a Markov blanket (Friston, 2013, 2019), suggesting that a boundary between system and environment arises naturally from this sparse structural connectivity as in directed acyclic graphs, without considering functional dynamics. Recent works have refined this argument, and justify a similar equivalence of Markov blankets and structural connectivity under an asymptotic approximation to a weak-coupling equilibrium (Friston et al., 2021b, see Eq. S8 in Supplementary Material). Under this assumption, recent works have predicted that Markov blankets will be commonplace in adaptive systems, e.g., in brain networks (Hipólito et al., 2021, Friston et al., 2021a). 


\section{A Bayesian directed acyclic graph}
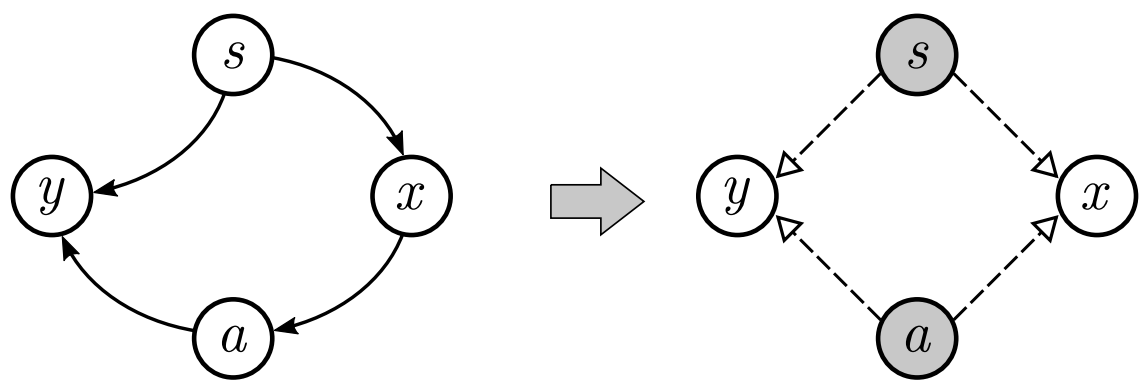

B Cyclic causal model
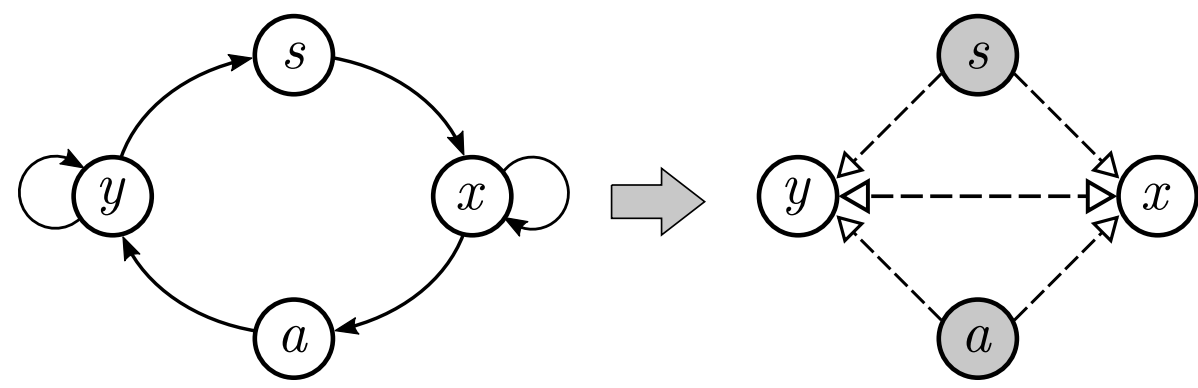

Figure 1: The left-hand figures show the structural connectivity of directed graphs. The right-hand figures show the conditional functional couplings of the system when the state of the 'blanket' $s, a$ is fixed. In directed acyclic graphs (A), the structural and functional couplings are directly related, and fixing the boundary results in conditional independence of $\mathrm{x}, \mathrm{y}$, yielding a Markov blanket. In directed cyclic graphs $(\mathbf{B})$, the recurrent structural connections result in additional functional couplings between variables, generating a new coupling between $x, y$ that 'crosses' the boundary, therefore not resulting in a Markov blanket in general.

In a recent article Aguilera et al. (2021), we studied analytically the existence of Markov blankets in nonequilibrium linear systems with recurrent connections. In these systems, their cyclic, asymmetric structure propagates reverberant activity system-wide, generating couplings beyond their structural connectivity (e.g. Fig. 11B). As a consequence, for most parameter configurations of a system, the sparse connectivity of the local Markov condition does not result in a Markov blanket. That is, even if a system only interacts with the environment via a physical boundary (e.g., a cell membrane or a perception-action interface), it will in general not display the conditional independence associated with a Markov blanket, a crucial issue that has been ignored in the FEP literature until very recently (Biehl et al., 2021; Aguilera et al., 2021).

These results raise fundamental and primary concerns about the frequent use of Markov blankets, not only in the FEP, but more generally as an explanatory concept for natural phenomena. A recent article (Friston et al., 2021a) has suggested that additional conditions (a sparsity of solenoidal couplings, a type of dynamical flows driving systems out of equilibrium) guarantee the emergence of Markov blankets, our study shows that these additional conditions become even more unlikely in the presence of recurrent connectivity in the studied nonequilibrium dynamics (Aguilera et al. 2021). It is important to note though these studies were restricted to linear systems and the generalization of these conclusions to nonlinear systems is yet to be studied. However, one could expect that nonlinear interactions might create a larger gap between intuitions drawn from structural considerations and actual functional couplings in the system.

These results do not imply that recurrent, nonequilibrium systems can never display Markov blankets. Our point, however, is that this only happens for highly specific cases, and certainly does not straightforwardly follow from the identification of a physical boundary, and it cannot be taken for granted that biological systems operate in this narrow parameter space. Such a finding would have significant implications for the physics of biological systems. Nevertheless, without evidence of this, debates about the implications of Markov blankets for living systems seem presumptuous and risks relegating the role of Markov blankets in elucidating the properties of living systems to the level of a potentially misleading metaphor rather than a verifiable hypothesis. 
Financial Support: M.A. is funded by the European Commission's under a Marie Skłodowska-Curie Action (grant agreement 892715). C.L.B. is supported by BBRSC grant BB/P022197/1.

\section{References}

Aguilera, M., Millidge, B., Tschantz, A., and Buckley, C. L. (2021). How particular is the physics of the free energy principle? Physics of Life Reviews.

Biehl, M., Pollock, F. A., and Kanai, R. (2021). A technical critique of some parts of the free energy principle. Entropy, 23(3):293.

Bruineberg, J., Dolega, K., Dewhurst, J., and Baltieri, M. (2021). The emperor's new markov blankets. Behavioral and Brain Sciences, page 1-63.

Friston, K. J., Da Costa, L., and Parr, T. (2021a). Some interesting observations on the free energy principle. Entropy, 23(8):1076.

Friston, K. J., Fagerholm, E. D., Zarghami, T. S., Parr, T., Hipólito, I., Magrou, L., and Razi, A. (2021b). Parcels and particles: Markov blankets in the brain. Network Neuroscience, 5(1):211-251.

Hipólito, I., Ramstead, M. J., Convertino, L., Bhat, A., Friston, K., and Parr, T. (2021). Markov blankets in the brain. Neuroscience \& Biobehavioral Reviews.

Pearl, J. (1988). Probabilistic reasoning in intelligent systems: networks of plausible inference. Morgan kaufmann.

Richardson, T. S., Spirtes, P., et al. (1996). Automated discovery of linear feedback models. Carnegie Mellon [Department of Philosophy]. 